\title{
A Note on the Physiology of Sex and Sex-determination.
}

\author{
By
}

\author{
J. H. Orton, D.Sc.,
}

Chief Naturalist at the Plymouth Laboratory

\section{CONTENTS.}

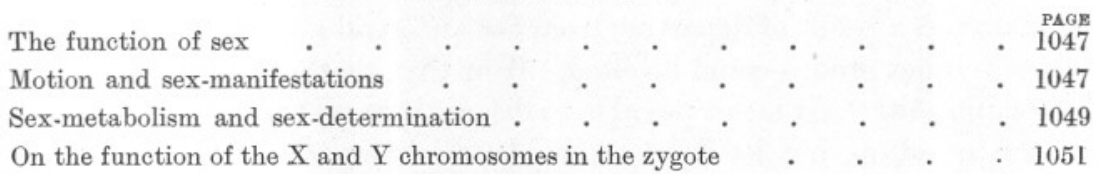

IN a previous paper in this Journal (Vol. XIV, p. 967) a theory of the control of sex in the oyster by rhythmical changes in the general metabolism has been outlined and discussed briefly with the information at present available. As the connexion between metabolism and sex has long been regarded as important, and by some, e.g. Geoffrey Smith (1913), as all-important, this relation may be considered briefly with regard to modern views on the physiology of sex.

\section{The Function of Sex.}

The function of sex is to provide a special means for the reproduction of the organism, therefore the relation of the variety of manifestations of sex to the correlated modes of reproduction in species is the true physiology of sex, which is clearly, though paradoxically, a biological problem; for it is not an unreasonable assumption that there is a general significant relation between sex-manifestations and particular needs in reproduction.

\section{Motion and Sex-manifestation.}

In a review of sex-phenomena in the animal kingdom as a whole, it is a fact that sedentary and parasitic and to a less extent sluggish forms are mainly hermaphrodite, whilst active animals are preponderantly bisexual; thus it would appear that mode of life is significantly related to sex-manifestation, and that bisexuality is favoured or rendered possible by an active life, whilst hermaphroditism meets the needs of reproduction 
in sedentary or parasitic forms; or it might be inferred that hermaphroditism has to meet the needs of such animals when one considers the remarkable instances of complemental males not infrequently associated with hermaphroditism (Cirripedes, Copepods, and others): bisexual sedentary animals are mostly aquatic, and effect cross-fertilisation-and apparently thereby avoid hermaphroditism - by extruding their genital products generally freely into the water.

As an example of the generalisations just outlined may be given the case of Crepidula and Calyptræa. The group to which these animals belong has as a group character bisexuality, and consists of moderately active members; but Crepidula and Calyptræa are sedentary forms (having adopted this mode of life in the course of ages) and are hermaphrodite (having apparently been obliged to assume the condition of sexuality as a result of departing from the ancestral mode of life). Similar cases in other groups could be cited. How then do these animals become hermaphrodite? It is not possible to discuss here all the possible answers to this question, but let it be assumed -in conformity with the general phenomena of sex-that hermaphroditism is imposed upon these organisms by the mode of life, and that, as the characteristic of the mode of life is simply environmental immobility with uniformity in habits of the adult, special arrangements become necessary for the purposes of reproduction. In sexual reproduction in general, however, cross-fertilisation appears to be a fundamental requirement, which, in the case of hermaphrodite individuals, may be met by various devices of alternating sex-phases (protandry, protogyny, alternating-hermaphroditism). If then hermaphroditism and cross-fertilisation be imperative in a sedentary species, it is obvious that a great variety of sex-arrangements might be evolved from those of its bisexual ancestors to satisfy these demands, and no general statement is likely to cover them. In the case of species, which have recently acquired hermaphroditism, active complemental males might be expected, or, indeed, active non-feeding males and sedentary females. It would appear that hermaphroditism in sedentary and parasitic species results from the necessity of providing the maximum possibilities for cross-fertilisation, and also for egg-production in all the individuals of the species which attain the state of food-absorption. But as - so to speak - anything can be a male, that is, very little organisation or food-reserve is necessary to produce a male, and as all individuals possess the potentiality to become male or female, it can be readily understood that an early general male phase should occur in the life-history followed by a female phase in the adult food-absorbing stage. In the latter stage there is every reason to believe that the fundamental metabolic processes are necessarily different from those existing in a male phase. It might indeed be argued that the type of metabolism imposed 
upon the adult stages in sedentary and parasitic animals is such as to demand femaleness as the main sex-phase, and that this is frequently one main cause of hermaphroditism.

Evidence is accumulating of a general difference in metabolism in males and females in bisexual species of animals, and many cases are now known where a change in metabolism results in a change of sex; the most striking of such cases being the change in the secondary-and the incomplete change in the primary-sexual characters in the spidercrab, Inachus, as a result of infection by Sacculina, shown by Geoffirey Smith (Q.J.M.Sc., 59, 1913).

\section{Sex-metabolism and Sex-determination.}

The literature on sex abounds with a recognition of the importance of particular types of metabolism associated with maleness and femaleness. (Goldschmidt, in Mechanism and Physiology of Sex-determination, 1923, gives the more important ones.) It would appear therefore that differential male and female metabolism occurs generally within a bisexual species, and the ground is prepared for the recognition of a cyclical or rhythmical change of metabolism during the life of the individual in certain hermaphrodites, and special conditions in those hermaphrodites in which eggs and sperm develop simultaneously-if, indeed, any such cases actually occur.

In recent investigations on sex-change in the Oyster $(O$. edulis $)$ and Crepidula fornicata, in both of which sex-change occurs-rhythmically and alternating in the former, and once in succession protandrically in the latter-the importance of metabolism in the sex-change has been impressed upon me. In Crepidula fornicata the duration of the male phase depends upon the capacity of the individual to function as a male (Orton, Nature, Vol. 110, 1922, p. 212), and may vary from about two to seven years. The conclusion to be drawn from this fact is that in this species the successful emission of sperm reacts on the general metabolism for the production of more sperm, and conversely, the failure to utilise sperm results in the cessation of a male metabolism, and the onset of a female type with production of eggs in the gonad. It follows, therefore, that sex, after the attainment of the initial male phase, is controlled in this case by some factor which concerns the whole organism-such as an accumulation of characteristic male reserve products-rather than by the production of a particular sex-hormone, which when it is formed runs a set course. In this case, therefore, the factor governing sex is that which calls forth the sex-hormones, if indeed sex-hormones-of the existence of which in this and other similar animals we have as yet no direct evidence-are produced. The information available on the factors controlling sex in 0 . edulis have been 
discussed in the Journ. Mar. Biol. Assoc., Vol. XIV, p. 1039, where it has been suggested that there occurs a metabolic rhythm controlling sex in two phases, in one of which protein metabolism is predominant and results in the production of eggs in the gonad, while in the other carbohydrate metabolism is paramount with the production of sperm in the gonad. Experimental evidence is now available that $O$. edulis does pass successively through the phases female, male, female, male, and indirect evidence that this sequence is repeated, but to an unknown extent (Orton, Nature, Vol. 114, p. 191, 1924). It is therefore further suggested that the production of carbohydrate products - especially glycogen -in excess of a certain concentration may be the stimulus, on the one hand, which causes the metabolism to begin in the protein phase with a consequent production of eggs in the gonad, while, on the other hand, the existence in the body of unusable protein - as at the phase immediately following egg-spawning-causes the metabolic rhythm to swing back to carbohydrate production with consequent sperm-formation in the gonad, and that this cycle of changes may be repeated several times. From the results of examining over 1,000 oysters which had previously spawned as females, there is clear evidence that the change from femaleness to maleness takes place normally within about twentyfour hours, usually much earlier but sometimes later, after spawning, and there is evidence that oysters in mass change over from protein to carbohydrate metabolism after spawning as females. In these sexchanges it may be assumed that separate generations of gonocytes take part in each sex-phase, and that the metabolic rhythm is a property of the organism.

These suggestions are made as an alternative to the ordinary sexhormone theory and have much to recommend them. The idea of a particular type of metabolism being associated with, if not controlling sex, has been accepted, or favourably entertained, by many biologists in the past, and is one which in recent times even Goldschmidt is reluctant to relinquish (1923, passim).

The theory of sex-control in the oyster, outlined above, and arrived at independently, is similar to that arrived at by Geoffrey Smith in his work on spider-crabs parasitised by Sacculina (1913). The theories which assume the type of metabolism to be of paramount importance in the control of sex are, however, generally regarded (e.g. Goldschmidt, 1923) as superseded by the sex-chromosome and sex-hormone theory. If, however, it is possible to reconcile the two theories, ancient and modern, and at the same time obtain a better general explanation of sex-phenomena, it is probable that progress is being made. 


\section{On the Function of $\mathrm{X}$ and $\mathrm{Y}$ Chromosomes in the Zygote.}

The modern view is that sex is determined in the zygote, and by combinations, or the absence, of the peculiar $\mathrm{X}$ and $\mathrm{Y}$ chromosomes. A very large proportion of sex-phenomena-though by no means allare satisfactorily explained on these lines, but it is submitted that a wider explanation of sex-phenomena may be obtained by incorporating the old idea of the importance of metabolism. It is suggested, therefore, that the $\mathrm{X}$ and $\mathrm{Y}$ chromosomes when present have the function of superimposing on the general metabolism of the species a metabolism of a particular type to which the gonad responds by producing eggs or sperm. On this view, therefore, all gonocytes have the potentiality (as is recognised by Goldschmidt) of becoming either oocytes or spermatocytes. On the sex-chromosome theory the gonad must (strictly) be assumed in certain cases to be either solely egg-producing or solely spermproducing, and when such an organism produces gonocytes of the opposite kind to that expected - as in Smith's crabs, the Gipsy moth, and many other cases - special strain has to be put on the sex-chromosome theory to bring it into line. It is certainly significant that in those cases in which the sex-chromosome theory - in its present formbreaks down - as in those cases in which occurs either an assumption of hermaphrodite characters, or a change of sex, or a heterosex condition - there is evidence of a change in the metabolism of the individual, as in Inachus parasitised by Sacculina and similar cases, Orchestia and similar cases, middle males in Crustacea, and in the remarkable alternative sex-condition in Bonellia and similar cases ; all of which are easily understood in terms of metabolic control, in the same way as the well-known sex-phenomena in bees, Cladoceras, and rotifers. Finally, the sexphenomena in the remarkable geographical races in Lymantria and frogs, in the crossing of which the $\mathrm{X}$ and $\mathrm{Y}$ chromosomes functions are turned topsy-turvy, are explained with less difficulty if the races be visualised as having developed different sex-metabolisms, which in their ontogenesis lose sex-specificity on "hybridisation." The case of Lymantria undoubtedly points to the function of the so-called sex-chromosomes being that of producing an environment for a sex rather than the sex itself. In fact, Lymantria and frogs provide us with what we have so long been looking for, namely, definite and measurable physiological variation within the species.

It is interesting to observe how such difficulties, as are mentioned above, are explained on the assumption that sex is controlled by special chromosomes. Goldschmidt (loc. cit.) confronts these difficulties in a definite manner, and has also perceived the undoubted correlation between differential metabolism and differential sex, as may be inferred from the 
following quotations. In the first place, in a review of the basic facts (1923, p. 138) the conclusion is drawn that "Every developing individual of a bisexual organism (species) contains the substances whose action can call forth the one or the other sex." From this broad generalisation alone it is permissible to consider whether sex may be controlled by the general nature of the metabolism--oriented by the peculiar chromosomes-or that these chromosomes themselves directly control sex. Again, in considering cases of successive hermaphroditism - such as that of the oyster (O. edulis)-(p. 180), "In regard to the conditions underlying the differentiation of both sorts of germ cells we know at least a very little. Both Ancel* and Buresch $\uparrow$ have shown that the same primordial germ cells (in Helix) may become eggs or spermatozoa, according as they enter into relations with so-called nurse-cells or not. What this means physiologically we do not know. An indication is given perhaps by the following. Wet were able to show that the differentiation of a spermcell into a spermatozoon was to a certain extent controlled by osmotic conditions whose regulation was a function of the follicle cells-and these are probably related physiologically to the nurse-cells of the eggs." ..." The conception of special quantitative conditions or reaction conditions of the sex-enzymes does not suffice as an explanation (of these cases). The special physiological condition with its localised mosaic-like or temporal variations, must rest somehow on a peculiarity of genetic constitution which up to date is both mechanically and physiologically unknown. Nor is it explained by the phrase - Factor for Monœcism." From this quotation and from others which could be given, it is clear that Goldschmidt recognises difficulties in the chromosome sex-control theory and is not afraid to demonstrate them. In dealing with intersexuality developed through parasitic castration, he remarks: "Now there can be no doubt that these facts (the transformation of secondary and primary sexual characters induced) are of great significance from the point of view of the sex problem, but their analysis has not in our opinion progressed far enough to permit of definite conclusions being drawn from them . . . for if the point of view advanced below turns out to be correct, there would be a good prospect of fitting in the keystone to the physiology of sex-determination." Then after discussing Geoffrey Smith's work on Sacculina and his theory of metabolic stimuli, Goldschmidt continues: "Nevertheless we believe there is a nucleus of truth in Smith's idea when freed from the exaggerated criticism of the hormone theory." The present writer here is in full agreement with Goldschmidt, who, following up the same subject, remarks that " The facts previously considered have brought us to the point where the hormones of sex-differentiation com-

\footnotetext{
* Ancel, P. Arch. Biol., 19. $1903 . \quad+$ Buresch, J. Arch. Zellf., \%, 1912.
} ‡ Goldschmidt, R. Arch. Zellf., 14, 1917. 
mence their activity. In regard to the mode of action of these bodies we have so far formulated no idea. Naturally there is nothing mystic about it, it is a chemical process and more than likely after all an action of the general metabolism. The action of the hormones probably calls forth a specific type of metabolism, and this is the ultimate and direct cause of the morphological differentiation of the sexes. If this were correct it would be the last word in the elucidation of the sex problem, and at the same time one which could be subjected to exact analysis."

The modified theory advanced herein that the $\mathrm{X}$ and $\mathrm{Y}$ chromosomes control sex-metabolism, and not strictly speaking sex itself-as by the direct production of sex-hormones-may indeed be regarded as one which has been tentatively accepted by Goldschmidt, as the preceding quotation shows. But Goldschmidt, having committed himself deeply to the sex-hormone theory, rejects G. Smith's theory of metabolic stimuli in calling forth sex-characters, although his written words show that he does not reject the underlying fundamental idea. It is obvious from the quotations given above that the views which have developed from the sex-chromosome theory are ripe for a change in the direction first advocated definitely by Geoffrey Smith, and it is probable that just as the latter may have underestimated the importance of hormones, so the sex-chromosome adherents may have erred in the opposite direction. It may not be necessary always to look for either a sexual formative substance or a specific sex-hormone; hence there is no need to despair if, for example, sanguinary transplantation experiments do not always give the result that certain sex-theories demand. The stimulus for the production of particular germ cells may vary greatly in kind throughout the animal kingdom, and. moreover, may vary in such a way that the definition of a sex-hormone may become merely a very general statement.

So far the theory of control of sex by special metabolic conditions has been discussed in relation to invertebrates mainly, but there are clear indications that the method of control of sex in animals has undergone evolution in the same way as other physiological processes and morphological characters. Experiments on transplantation of the gonad and/or injection of gonadial secretions show that in birds and mammals, and possibly also Amphibia, the secondary sexual characters are under the control of the gonadial system, while in invertebrates (and probably many cold-blooded vertebrates) the gonad has no such control. Thus in birds and mammals the gonad controls secondary sexual-characters in the same way as the thyroid controls growth, that is to say, the control of sex-function, once the sex has become fixed, has passed to a subsidiary centre. In this respect it seems probable that the acquisition of a definite bodily temperature has rendered easy the introduction of a decentralised 
control of sex-characters-and, indeed, other bodily functions-and in any case the importance of external physical conditions has become no longer of such great importance on the general effect on metabolism, as in cold-blooded animals, since all the enzymes in the body can function in a constant or almost constant temperature, being subjected only indirectly to the influence of external conditions.

Thus, in the warm-blooded vertebrates, birds and mammals, sexpotentiality - in terms of the theory outlined above-is determined primarily by the metabolic potentialities (which may, however, be delegated in highly developed forms to a single sex-hormone) of the zygote (in its chromosome constitution), but this sex-potentiality is modifiable at an early stage or later by the secretions of the early developing gonad which takes over to a large - but uncertain - extent the directing influence of the general metabolism. On the other hand, in invertebrates the control of both the primary and sexual characters would appear to lie in the general organisation of the organism as determined primarily by the metabolic potentialities of the zygote (from its chromosome constitution), but modifiable by a fundamental change in the metabolism during the life of the individual. With regard to monœcious forms in invertebrates, it follows that if the theory outlined is tenable, rhythmical or cyclical variation in metabolism correlated with sex-phases, as appears to be the case in $O$. edulis, should always occur, and a field for research is indicated. Cyclical change in the production of different sex-forms correlated with metabolic changes is known to occur in some species over a number of generations, so that the concentration of these changes in a single monøecious individual is merely a difference of distribution of life-phases in time, and affords one more instance of the fascinating adaptability of the organism in sex-contrivances.

The theory that sex is controlled by a particular type of metabolismwhether this be produced by bio-physico-chemical events set in motion by $\mathrm{X}$ and $\mathrm{Y}$ chromosomes, or by the imposition of a particular mode of life on the individual - is in harmony with sex-phenomena in gross in the animal kingdom, in which the active species have generally males and females with different organisations and functions to perform correlated in many (and probably most) cases with a different general metabolism, while sedentary and parasitic forms in their adult anabolic stage are mainly either wholly female or partly female according to the possibilities for fertilisation in particular forms.

The theory of sex-control by a type of metabolism normally unfolded by $\mathrm{X}$ or $\mathrm{Y}$ chromosomes enables us to consider these latter entities as of essentially the same nature as the autosomes, but with the function of adding to a stable unit, which we may call the specific neutral soma, the peculiarities of sex. Non-sexual characters inherited with sex would 
therefore be generally expected to have their determinants wholly or in part in the $\mathrm{X}$ or $\mathrm{Y}$ chromosome.

Since the function of sex is to reproduce the organism, the mode of reproduction employed in particular cases must be of greater importance than the actual process of sex-control, and will determine the nature and incidence of sex-control. Sex-control is, therefore, a matter of secondary importance in a broad consideration of sex-phenomena, and it would appear that these can only be properly understood as a whole, and indeed in particular cases, when considered in relation to phylogeny and bionomics. 
